\title{
Glidewire-assisted Foley catheter placement: a simple and safe technique for difficult male catheterization
}

\author{
Rei K. Chiou, MD; Himanshu Aggarwal, MD; Wen Chen, APRN
}

\begin{abstract}
Difficulty in Foley catheter placement is a frequently encountered problem. We describe a simple and safe technique for this condition. Rather than using force, which may lead to the formation of a false passage, one should place a glidewire into the bladder through the area of resistance, followed by the placement of a Foley catheter over the glidewire. This is a very easy procedure and can be taught to nurses and nurse practitioners to avoid an unnecessary call for a urologist in the emergency department.
\end{abstract}

Can Urol Assoc J 2009;3(3):189-92

\section{Résumé}

Il arrive fréquemment que des difficultés surviennent durant l'installation d'une sonde de Foley. Nous décrivons ici une technique simple et sûre pour remédier à la situation. Plutôt que d'utiliser la force, qui peut entraîner la formation d'un faux passage, nous conseillons d'insérer un fil-guide dans la vessie par la zone de résistance suivi de l'installation de la sonde de Foley par-dessus le fil-guide. Cette intervention est très facile à réaliser et peut être enseignée aux infirmières et aux infirmières praticiennes en cas de difficulté lors de l'insertion de la sonde et ainsi éviter de devoir faire venir un urologue à la salle d'urgence.

\section{Introduction}

The placement of a Foley catheter is a common procedure and an essential skill for nurses..$^{1-3}$ Difficulty in placing a Foley catheter is a commonly encountered problem among nurses and nurse practitioners. When one encounters difficulty in placing a Foley catheter using a regular technique, it has been recommended to use a reinforced tip catheter, such as a Coudé tip catheter for patients with benign prostatic hyperplasia (BPH). ${ }^{4}$ It is also a common tendency to push harder when one encounters difficulty in placing a Foley catheter. However, in the presence of a urethral stricture, the use of excessive force or reinforced catheters increases the risk of false passages. If a false passage is created, patients may require a more complicated endoscopic procedure or suprapubic catheter placement.
We describe a simple and safe technique that we have taught to several nurse practitioners, urology nurses and non-urology specialized physicians to successfully place Foley catheters in difficult cases. This technique can help avoid unnecessary emergency department visits for urologists. This technique has been used in more than 150 cases of difficult catheterization without any complications.

\section{Technique}

When encountering difficulty in placing a Foley catheter in a male patient, one should ensure that the urethra is lubricated adequately. This can be better achieved by instilling lubricants into the urethra rather than solely dipping the tip of the catheter in the lubricant. If the patient experiences substantial pain during a Foley catheter placement, the intraurethral instillation of topical anesthetics ( $2 \%$ xylocaine jelly) is also helpful. This alleviates pain and decreases sphincter contraction that creates resistance to the catheter placement.

If, after these manoeuvres, one cannot place the Foley catheter, then a glidewire-assisted technique should be used rather than forcefully pushing the Foley catheter. Examples of such a glidewire are the angled tip, 0.038 inches, $150 \mathrm{~cm}$, No. 630-103 wire (Boston Scientific Inc.) or the straight tip, 0.038 inches, $150 \mathrm{~cm}$, No. HW-038150 Hi-wire (Cook Medical Inc.). Glidewires and Hi-wires are hydrophilic coated smooth surface wires that have very soft ends, which makes it safe to pass through a strictured or distorted urethra with minimal risk of injury. When a hydrophilic glidewire comes in contact with water it becomes slippery and the Foley catheter can easily be slid around it because of its smooth and wet surface. The glidewire comes in different sizes and lengths. We commonly use a 0.038-inch glidewire with $150 \mathrm{~cm}$ length for this technique.

After moisturizing the glidewire with sterile water by injecting water into the plastic sheath that contains the glidewire and then using a wet sponge to keep the glidewire moist during use, the soft floppy end of the glidewire or $\mathrm{Hi}$-wire (not the stiff end of the wire) should be placed into 
the urethral meatus and then gently advanced to pass through the urethra into the bladder. If there are no false passages, this soft and slippery wire can easily be placed up to the level of the bladder negotiating even a very tight stricture or distortion of the urethra.

The next step is to use a No. 10 blade to cut a slit on the Foley catheter tip to expose the lumen (Fig. 1). Care must be taken to avoid cutting the lumen that leads to the balloon. Testing leakage of the balloon after cutting is recommended. The Foley catheter can then be placed around the wire through the stiff outer end of the glidewire. After that, the Foley catheter can be advanced through the urethra into the bladder (Fig. 2). With the glidewire in place, one can push somewhat harder, in case resistance is met, when passing the Foley catheter because the passage has already been established with the glidewire.

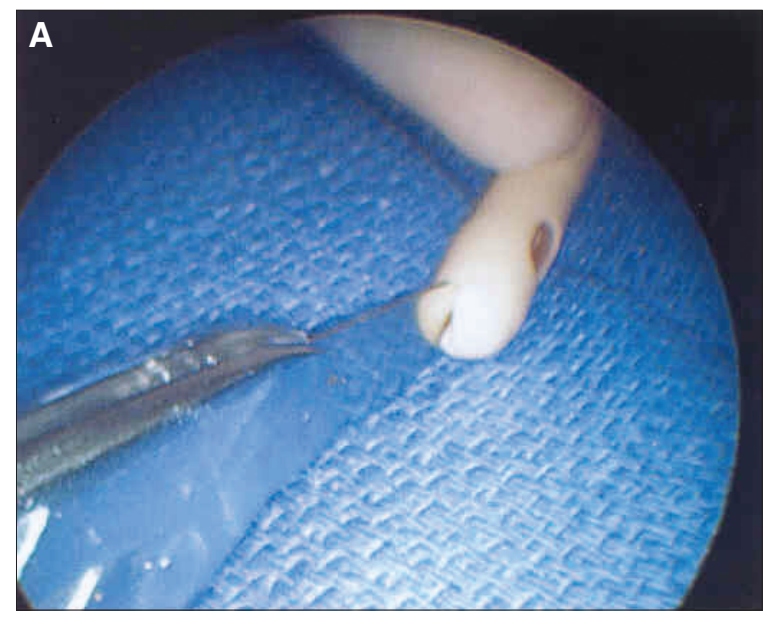

Fig. 1. Use of No. 10 blade to cut a slit on the tip of the Foley catheter (A). The lumen of the Foley catheter is exposed (B).
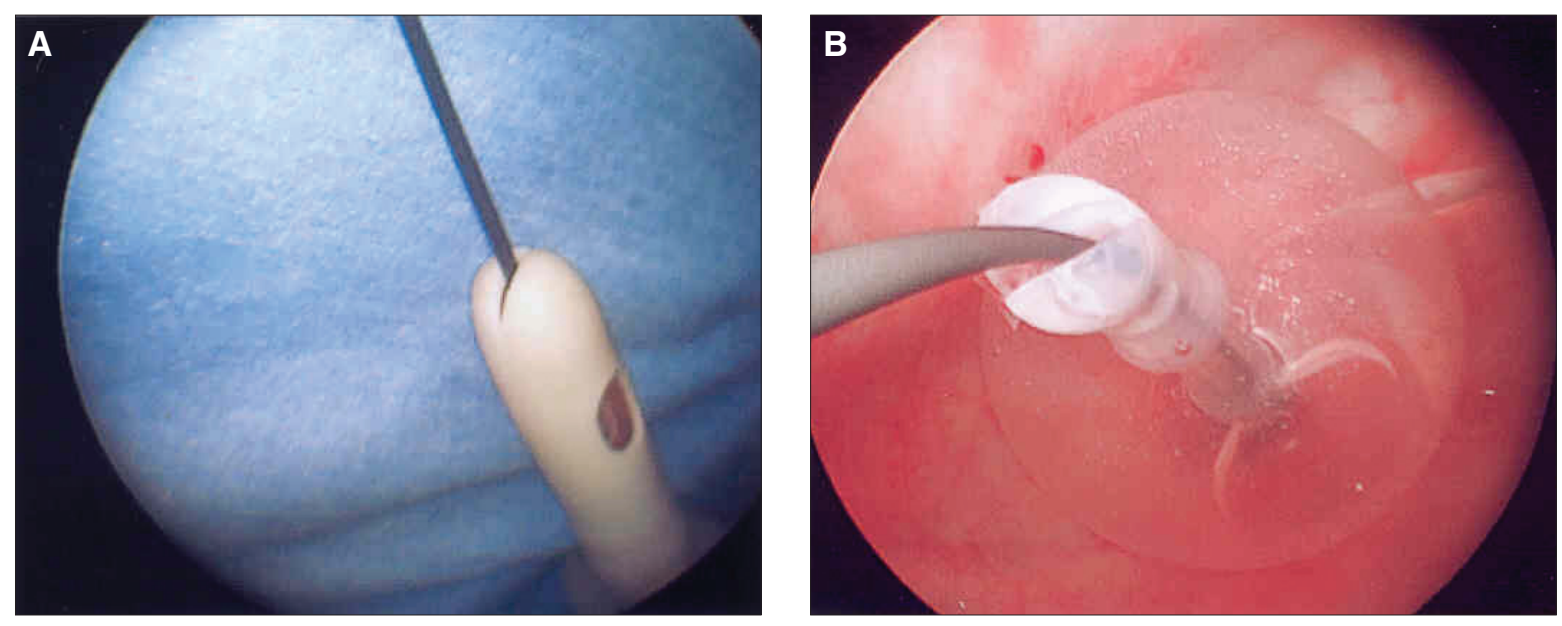

Fig. 2. Placement of the Foley catheter around the wire through the stiff outer end of the glidewire (A). The Foley catheter is advanced through the urethra and into the bladder (B). 
procedure for urinary retention. Difficult catheterization can be very troublesome in inexperienced hands in the absence of the proper technique. The underlying causes of difficulties include poor lubrication, pain (leading to sphincter contraction and resistance), meatal stenosis, urethral stricture, urethral distortion, distortion of prostatic urethra due to prostate enlargement or surgery, or bladder neck contracture. When encountering difficulties, first of all one should ensure adequate lubrication of the urethra because solely dipping the tip of the Foley catheter in lubricant may not be adequate. Instillation of lubricant into the urethra is always better (most Foley catheter trays have a syringe of lubricant that can be used to instill lubricants into the urethra). If the patient has pain during an attempt to place the Foley catheter, there may be tightening of sphincter muscles against the catheter. Instillation of a topical anesthetic ( $2 \%$ xylocaine jelly) inside the urethra may help with this condition. If the catheter cannot be placed after adequate lubrication, one must assess the underlying causes of the problem.

On initial diagnosis, meatal stenosis is easily identifiable, and the catheter cannot be placed into the stenotic meatus. In such cases meatal dilatation needs to be performed. Other than meatal stenosis, the main differential diagnosis for difficult catheterization in a male patient is a urethral stricture or a prostate problem. Most urethral strictures in patients without any prior prostate surgery are at the pendulous or bulbous urethra. By simply knowing where the catheter meets resistance, one should be able to determine the underlying cause of the problem. If the catheter stops at the penile, scrotal, or perineal levels, it is probably stricture. If the catheter hits the level of the prostate (beyond the urogenital diaphragm or the perineal level), but cannot pass through, it is probably caused by a prostate problem. In the absence of prior prostate surgery, the resistance at the prostate level usually results from $\mathrm{BPH}$ or a bulky prostate cancer. In patients who have had prior prostate surgery, the obstruction is usually due to bladder neck contracture.

After encountering difficulty in placing a regular Foley catheter, a Coudé tip catheter can be used in patients with $\mathrm{BPH} .{ }^{4}$ With adequate lubrication and keeping the curved Coudé tip pointing upwards, one can negotiate the distorted prostatic urethra and elevation of the bladder neck created by BPH. However, a Coudé tip catheter should only be used in patients with prostate problems, and not in cases of urethral stricture. If a patient has a urethral stricture, the use of a Coudé tip catheter can create a false passage as the catheter tip points toward the side. The strictured lumen of the urethra is usually very resistant to passage of a Coudé catheter. A Coudé tip catheter usually cannot achieve the goal of dilating the stricture and tends to pierce the delicate urethral wall at the side of the strictured lumen, creating a false passage. The glidewire-assisted technique that we described is useful both in patients with urethral strictures and prostate problems. It also minimizes the risk of a false passage. If the patient has a history of prostate surgeries such as transurethral resection of the prostate for $\mathrm{BPH}$ or radical prostatectomy for prostate cancer, the common cause of obstruction is usually a bladder neck contracture or vesicourethral anastomotic stricture, respectively. The glidewire-assisted technique is still appropriate and useful in these patients.

Some early studies have recommended flexible cystoscopy by a urologist after initial failed catheterization to evaluate the exact pathology and passage of a glidewire into the bladder under direct vision so that a Foley catheter can be passed around it. ${ }^{5}$ However, in our experience and in other studies $^{6}$ the blind use of the glidewire can be safely attempted as a second-line treatment, and this is very effective in most of the difficult cases of catheterization, including urethral stricture and prostatic problems. The technique we described can be easily and safely used by any trained nurse or a family physician in peripheral hospitals. If even the glidewire cannot be placed, a flexible cystoscopy should be performed by a urologist to diagnose underlying pathology and to evaluate for the need of suprapubic catheterization or urethral dilation. Also in cases of intraoperative difficulty in placing a Foley catheter, urethroscopy or endoscopy can be performed to evaluate the need for suprapubic tube placement of urethral dilation.' If a urethral dilation is required, we prefer to carry out a balloon dilation around the glidewire. The Foley catheter can then be placed around the glidewire using the technique we described.

We also recommend watching for any urethral bleeding during the initial attempt of Foley catheter placement, as this usually indicates some degree of urethral tear or false passage. One should stop any further attempt on the first sign of false passage and call for the help of a specialist because a false passage complicates further management.

\section{Conclusion}

When encountering difficulty in Foley catheter placement in a male patient, we recommend a simple and safe technique of a glidewire-assisted Foley catheter placement. This technique minimizes the risk of a false passage that is created by forcing catheters or using Coudé tip catheters in case of urethral stricture. This is an easy, safe and very effective procedure that can be taught to nurses or family practioners and can avoid unnecessary emergency calls for urologists, especially in peripheral hospitals. 
From the Creighton University Medical Center, Division of Urology, Department of Surgery, Omaha, Nebr.

Competing interests: None declared.

\section{References}

1. Hardy J. Urinary catheterisation in male patients. Nurs Stand 2006;21:59.

2. Robinson J. Urethral catheter selection. Nurs Stand 2001;15:39-42.

3. Doherty W. Male urinary catheterisation. Nurs Stand 2006;20:57-63.

4. Clinical Practice Guidelines Task Force, Society of Urologic Nurses and Associates. Male urethral catheterization. Urol Nurs 2006:26:315.

5. Krikler SJ. Flexible urethroscopy: use in difficult male catheterisation. Ann R Coll Surg Engl 1989;71:3.

6. Villanueva $C$, Hemstreet GP III. Difficult male urethral catheterization: a review of different approaches. Int Braz I Urol 2008;34:401-11.

7. Jordan GH, Winslow BH, Devine CJ Jr. Intraoperative consultation for the urethra. Urol Clin North Am 1985; 12:447-52.

Correspondence: Dr. Rei K. Chiou, Professor and Chief, Division of Urology, Creighton University Medical Center, 601 North 30th St., Suite 3700, Omaha NE 68131; fax: 402 280-4309; ReiChiou@chiwest.com

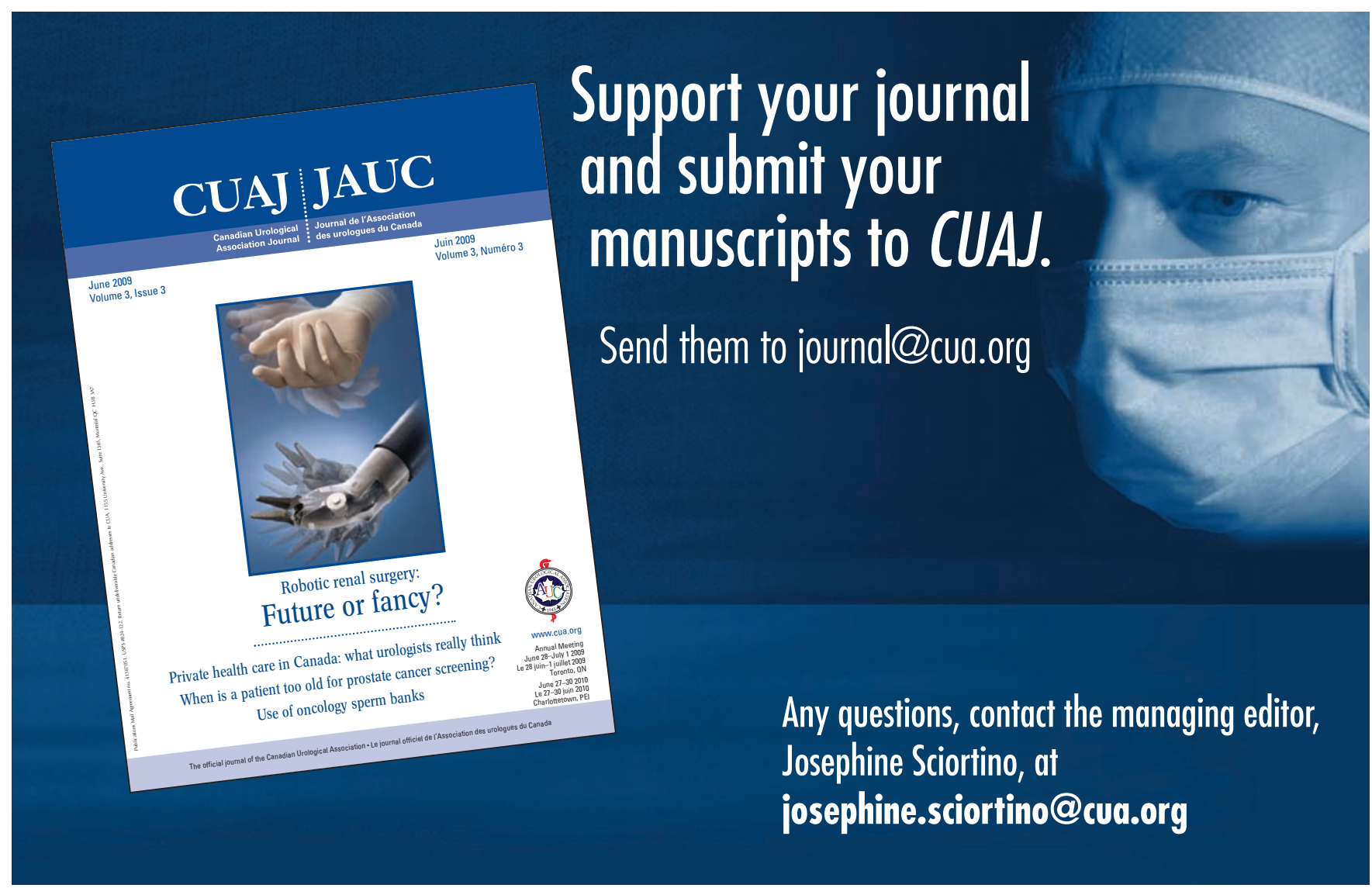

